MATEC Web of Conferences 8, 02002 (2013)

DOI: $10.1051 /$ matecconf $/ 20130802002$

(c) Owned by the authors, published by EDP Sciences, 2013

\title{
Engineering materials for mid-infrared optical sensor applications
}

Richardson, K. A ${ }^{1}$., Musgraves, J. D ${ }^{1}$., Wachtel, P. ${ }^{1}$, Novak, S. ${ }^{1}$,Danto, S. ${ }^{1}$, Agarwal, A. ${ }^{2}$, Singh, V. ${ }^{2}$, Lin, P-T ${ }^{2}$, Kimerling, L.C. ${ }^{2}, \mathrm{Hu}, \mathrm{J}^{3}$, Yi, Z. ${ }^{3}$, Lin, H. ${ }^{3}$, Giammarco, J. ${ }^{4}$, Soliani, A.P ${ }^{4}$, Luzinov, I. ${ }^{4}$, Hensley, J. ${ }^{5}$

${ }^{1}$ Townes Laser Institute, CREOL, College of Optics and Photonics, Univ. Central FL, Orlando FL

${ }^{2}$ Microphotonics Center, Massachusetts Institute of Technology, Cambridge MA

${ }^{3}$ Department of Materials Science and Engineering, University of Delaware, Newark, DE

${ }^{4}$ School of Materials Science and Engineering, Clemson University, Clemson, SC

${ }^{5}$ Physical Sciences Inc., Andover, MA

\begin{abstract}
Planar optical structures based on functionalized chalcogenide glasses provide a superb device platform for chemical and biological sensing applications. Chalcogenide glasses have demonstrated promise as materials for infrared sensing as they exhibit transparency over a large range of infrared wavelengths and tunable optical properties through doping and/or compositional tailoring. Waveguides, resonators and other components processed on-chip (silicon, Si) can be realized such that the strong enhancement in the electromagnetic field confined within a high index contrast resonator, leads to highly sensitive photon-matter interactions in a small footprint. In this paper we discuss the development of highly sensitive chalcogenide glass based microdisk resonator sensors that measure resonant peak shifts caused by refractive index change upon exposure to a chemical analyte. The specificity of the microdisk resonator sensors is enhanced by applying specialized polymer films and nanofoams that respond in a predictable fashion when exposed to a chemical analyte of interest. Discussed are key material science challenges needed to enable highly sensitive and specific sensors based on such complex multi-material assemblies and the fabrication issues that ultimately define resulting optical performance.
\end{abstract}

This is an Open Access article distributed under the terms of the Creative Commons Attribution License 2.0, which permits unrestricted use, distribution, and reproduction in any medium, provided the original work is properly cited. 